\title{
Front Matter: Volume 11175
}

, "Front Matter: Volume 11175," Proc. SPIE 11175, Optifab 2019, 1117501 (15 November 2019); doi: 10.1117/12.2559224

SPIE. Event: SPIE Optifab, 2019, Rochester, New York, United States 


\section{PROCEEDINGS OF SPIE}

\section{Optifab 2019}

Blair L. Unger

Jessica DeGroote Nelson

Editors

14-17 October 2019

Rochester, New York, United States

Sponsored by

SPIE

Cosponsored by

The American Precision Optics Manufacturers Association (United States)

Published by

SPIE

Volume 11175 
The papers in this volume were part of the technical conference cited on the cover and title page. Papers were selected and subject to review by the editors and conference program committee. Some conference presentations may not be available for publication. Additional papers and presentation recordings may be available online in the SPIE Digital Library at SPIEDigitalLibrary.org.

The papers reflect the work and thoughts of the authors and are published herein as submitted. The publisher is not responsible for the validity of the information or for any outcomes resulting from reliance thereon.

Please use the following format to cite material from these proceedings:

Author(s), "Title of Paper," in Optifab 2019, edited by Blair L. Unger, Jessica DeGroote Nelson, Proceedings of SPIE Vol. 11175 (SPIE, Bellingham, WA, 2019) Seven-digit Article CID Number.

ISSN: 0277-786X

ISSN: 1996-756X (electronic)

ISBN: 9781510630635

ISBN: 9781510630642 (electronic)

Published by

SPIE

P.O. Box 10, Bellingham, Washington 98227-0010 USA

Telephone +1 3606763290 (Pacific Time) · Fax +1 3606471445

SPIE.org

Copyright (c) 2019, Society of Photo-Optical Instrumentation Engineers.

Copying of material in this book for internal or personal use, or for the internal or personal use of specific clients, beyond the fair use provisions granted by the U.S. Copyright Law is authorized by SPIE subject to payment of copying fees. The Transactional Reporting Service base fee for this volume is $\$ 21.00$ per article (or portion thereof), which should be paid directly to the Copyright Clearance Center (CCC), 222 Rosewood Drive, Danvers, MA 01923. Payment may also be made electronically through CCC Online at copyright.com. Other copying for republication, resale, advertising or promotion, or any form of systematic or multiple reproduction of any material in this book is prohibited except with permission in writing from the publisher. The CCC fee code is 0277 $786 \mathrm{X} / 19 / \$ 21.00$.

Printed in the United States of America by Curran Associates, Inc., under license from SPIE.

Publication of record for individual papers is online in the SPIE Digital Library.

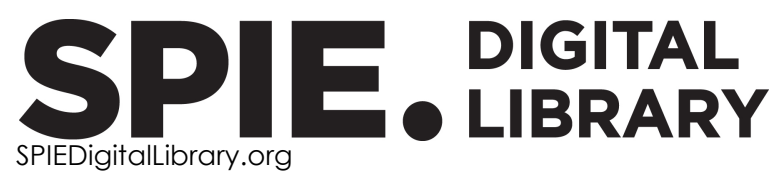

Paper Numbering: Proceedings of SPIE follow an e-First publication model. A unique citation identifier (CID) number is assigned to each article at the time of publication. Utilization of CIDs allows articles to be fully citable as soon as they are published online, and connects the same identifier to all online and print versions of the publication. SPIE uses a seven-digit CID article numbering system structured as follows:

- The first five digits correspond to the SPIE volume number.

- The last two digits indicate publication order within the volume using a Base 36 numbering system employing both numerals and letters. These two-number sets start with 00, 01, 02, 03, 04, 05, 06, 07, 08, 09, OA, OB ... 0Z, followed by 10-1Z, 20-2Z, etc. The CID Number appears on each page of the manuscript. 


\title{
Contents
}

\author{
vii Authors \\ ix Conference Committee
}

PLENARY SESSION

$1117504 \quad$ Apprenticeship: precision optics manufacturing technician (Plenary Paper) [11175-17]

ADVANCEMENTS IN CONVENTIONAL FABRICATION METHODS

1117505 Creating sub angstrom surfaces on planar and spherical substrates [11175-1]

1117506 A method to discriminate between upper and lower side material removal in double-side polishing [1 $1175-2]$

1117507 Three-dimensional configurable IC optic material for precision CNC optical polishing [1 $1175-3]$

1117508 Subsurface damage measurement of single crystal germanium and borosilicate glass BK-7 [11175-4]

11175 OA Particle distribution characterization on material removal uniformity in chemical mechanical polishing [1 $11175-6]$

\section{INTEGRATING AUTOMATION AND LASERS INTO OPTICS FABRICATION}

11175 OB Robotic polishing in asphere manufacturing [11175-7]

11175 OD Ultra precision glass machining through ultrasonic assisted diamond turning [11175-9]

$11175 \mathrm{OE} \quad$ High speed ultraprecision machining of germanium [11175-10]

11175 OF Micro-laser assisted single point diamond turning of fused silica glass [1 $1175-111]$

DETERMINISTIC PROCESSING OF OPTICS

$11175 \mathrm{OH} \quad$ Precision machining of strong aspheres made of calcium fluoride and fused silica [11175-13] 
11175 ol Material removal for small compressions on fused silica on an OptiPro Ultraform finishing machine [1 $1175-14]$

11175 OK Fabrication of continuous phase plates based on bonnet polishing [11175-16]

DETERMINISTIC FINISHING PROCESSES

$11175 \mathrm{OL} \quad$ Wavefront improvement by IBF-processed correction surfaces [11175-24]

$111750 \mathrm{M}$ Study on the performances of dwell time algorithms in ion beam figuring [11175-26]

$11175 \mathrm{ON} \quad$ Extending magnetorheological finishing to address short radius concave surfaces and mid-spatial frequency errors [1 $1175-28]$

1117500 Accounting for MRF spot removal rate variation caused by plunge depth deviation [1 $1175-30]$

11175 OP Polishing ultra-precision ground aspherical surfaces with MRF [1 $1175-32]$

$111750 Q \quad$ New meter-class MRF platforms offer multiple size and capability options [1 $1175-34]$

\section{OPTICAL ENGINEERING}

11175 OW Design, simulation and manufacturing a CFRP prototype mirror for active/adaptive optics [11175-23]

\section{FREEFORM MANUFACTURING}

$111750 Z$ Freeform testability considerations for subaperture stitching interferometry [11175-35]

1117510 Definitions of criteria for assessing feasibility and measurability of freeform surfaces [11 175-36]

$1117511 \quad$ Advances in freeform manufacturing [11175-37]

1117512 Near-conformal window assembly for airborne payloads: improved time on-station and optical performance [1 $1175-38]$

1117513 Measurement of form and mid-spatial-frequency errors of specular freeform surfaces [11175-39]

1117514 Scaling-up freeform manufacturing: challenges and solutions [11175-40] 
ADVANCES IN FREEFORM AND NOVEL METROLOGY TECHNIQUES

1117515 Advancements in non-contact freeform metrology with datum structures [11175-41]

1117516 Advantages of a low coherence interferometer for optical testing [11175-43]

1117517 Spectrally controlled interferometry for improved radius of curvature measurement [1 1175-44]

SURFACE ROUGHNESS AND OPTICAL ALIGNMENT

1117519 Sub-angstrom surface roughness metrology with the white light interferometer [1 $1175-46]$

11175 1B Rotation-free centration measurement for fast and flexible inspection of optical lens systems [11 1 175-48]

$111751 \mathrm{C} \quad$ Novel active alignment technique for measuring tilt errors in aspheric surfaces during optical assembly using Lens Alignment Station (LAS) [1 $1175-49]$

11175 1D Beyond centration: how to create, read, and use a datum system per ISO-10110-6 (2015) [1 $1175-50]$

MID-SPATIAL FREQUENCY AND WAVEFRONT ERROR MEASUREMENT METHODS

$111751 \mathrm{E} \quad$ Analysis of mid-spatial frequency errors in two dimensions at metal mirror fabrication [11175-51]

$1117511 \quad$ Measurement of a concave spherical mirror with $\mathbf{5 0} \mathbf{~ m m}$ radius of curvature by three dimensional nanoprofiler using normal vector tracing [1 $11175-55]$

\section{OPTICAL COATINGS}

$111751 \mathrm{~L} \quad$ Large-scale freeform surface ultra-thin film coating uniformity measurement based on a dynamic spectroscopic ellipsometer [11175-58]

1117510 Influence of ion assistance on optical properties, residual stress and laser induced damage threshold of HfO2 thin film by use of different ion sources [11175-61]

\section{STRUCTURED OPTICAL SURFACES}

$111751 \mathrm{P} \quad$ Microlens array based three-dimensional light field projection and possible applications in photolithography [1 $11175-62]$ 
11175 is Multi-functional immersion grating by homemade freeform cutting machine [11175-65]

$111751 \mathrm{U}$ Molded anti-reflective structures of chalcogenide glasses for infrared optics by precision glass molding [1 $1175-67]$

$111751 \mathrm{~V} \quad$ Approaches and methodologies for process development of thin glass forming [11175-68]

\section{POSTER SESSION}

$111751 Y \quad$ Silicone grating fabricated using photoresist mold [1 1175-71]

1117520 An effective way to calibrate the external errors which are contributed in the interferometric test for spherical surfaces [1 $1175-73]$

1117521 Experimental investigation on processing of fused silica microchannels by high repetition rate femtosecond laser [11175-74]

1117523 Study of thermal deformation monitoring system with long short term memory network in alignment turning system [1 $11175-76]$

1117526 Development of a reflective spiral phase plate based on MRF polishing [1 $1175-80]$

1117529 Study on wear behavior of grinding wheel for the generating process of UV grade fused silica [11175-83]

$111752 \mathrm{~B} \quad$ High precision interferometric measurement of freeform surfaces from the well-defined sub-aperture surface profiles [1 $1175-85]$

11175 2C Diamond turning of aluminum image slicers for astronomical applications [11175-86]

$111752 \mathrm{E}$ Compensation of thermal drift during the single-point diamond turning process based on the LSTM [1 $11175-89]$ 


\title{
Authors
}

Numbers in the index correspond to the last two digits of the seven-digit citation identifier (CID) article numbering system used in Proceedings of SPIE. The first five digits reflect the volume number. Base 36 numbering is employed for the last two digits and indicates the order of articles within the volume. Numbers start with 00, 01, 02, 03, 04, 05, 06, 07, 08, 09, OA, OB...0Z, followed by 10-1Z, 20-2Z, etc.

\author{
Auger, Hugues, 2C \\ Baghsiahi, Hadi, OW \\ Beier, Matthias, 1E \\ Bergs, Thomas, 1U, $1 \mathrm{~V}$ \\ Berkson, Joel D., 12 \\ Binkele, T., 13 \\ Blalock, Todd, 14 \\ Bodlapati, Charan, OE, OF \\ Brooks, David, OW \\ Brousseau, Denis, 2C \\ Brunelle, Matt, 14 \\ Bulla, B., OD \\ Chabot, Tristan, 2C \\ Chang, Jiun-Lee, 29 \\ Chang, Shenq-Tsong, 20 \\ Chen, Fong-Zhi, 23 \\ Chen, Hua-Lin, 20, 29 \\ Chen, Hung-Pin, 20, 29 \\ Chen, Jun-Cheng, 23 \\ Chen, Wei-Chun, 20 \\ Chen, Xian-hua, OA, OK \\ Coniglio, Jen, 14 \\ Da Costa Fernandes, B., 06 \\ Dambon, O., OD \\ DeFisher, Scott, 11, 15 \\ DeGroote Nelson, Jessica, 14 \\ DeMarco, Mike, 00 \\ Dembele, Vamara, $1 \mathrm{~L}$ \\ Deng, Wen-hui, OK \\ Doel, Peter, OW \\ Doetz, M., OD \\ du Jeu, Christian, 10 \\ Dumas, Paul, OQ \\ El Handrioui, Hassan, 10 \\ Endo, Katsuyoshi, 11 \\ Essameldin, M., 13 \\ Feldkamp, Roman, OL \\ Ferralli, Ian, 14 \\ Fleischmann, F., 13 \\ Fourez, Julien, 10 \\ Freischlad, Klaus R., 16 \\ Garden, Rognvald, $1 \mathrm{C}$ \\ Gemmill, William R., 07 \\ Gilles, Matthieu, 10 \\ Green, Mark, 1C \\ Gross, Herbert, 1E \\ Grunwald, Tim, 1U, 1V \\ Hahne, F., 1B \\ Hall, Chris, 00
}

\author{
Hartung, Johannes, $1 \mathrm{E}$ \\ Hashimoto, Kota, 11 \\ Heinisch, J., 1B \\ Henning, T., 13 \\ Hilbig, D., 13 \\ Hinrichs, Keith M., 12 \\ Huang, Chien-Yao, 23, 29 \\ Huang, Lei, OM \\ Hyun, Sang-Won, 26, 2B, 2E \\ Idir, Mourad, OM \\ Ikeda, Yusuke, 1 Y \\ lles, Shawn, 05, 19 \\ Je, Soonkyu, 2B \\ Jeon, Min-Woo, 26 \\ Jeong, Byeong-Jun, 2E \\ Jeong, Seok-Kyeong, 26, 2E \\ Jones, Martyn, OW \\ Kang, Di, OE, OF \\ Kang, Jong-Gyun, 26, 2E \\ Kang, Jungmin, 11 \\ Kim, Daesuk, 1L \\ Kim, Geon-Hee, 26, 2B, 2E \\ Knight, Terry, 07 \\ Koliopoulos, Chris L., 16 \\ Kraft, Nicholas, 07 \\ Lambropoulos, John C., 08, 0 I \\ Lammers, Tom, $1 \mathrm{E}$ \\ Lang, W., 13 \\ Langehanenberg, P., IB \\ Lee, Won-Kyun, 2E \\ Leibowitz, Noah, 01 \\ Li, Shugang, 10 \\ Liao, Defeng, OA \\ Liao, Kai, 21 \\ Lin, Wei-Cheng, 20, 23, 29 \\ Lin, Yu-Wei, 20 \\ Liu, Bin, 21 \\ Liu, Mincai, 10 \\ Liu, Zhichao, 10 \\ Lucas, Felix, $\mathrm{OH}$ \\ Luo, Jing, 10 \\ Lynch, Tim, 14 \\ Maloney, Chris, ON, OQ \\ Mandina, Mike, 04 \\ Martin, Tony R., 07 \\ Maunier, C., 06 \\ Mei, Xuesong, 21 \\ Mende, Hendrik, IV \\ Messner, William, ON
}


Murphy, Paul E., OZ

Myer, Brian, 14

Navare, Jayesh, $\mathrm{OE}, \mathrm{OF}$

Neauport, J., 06

Nelson, Jayson, 05, 19

Okura, Yukinobu, is

Olszak, Artur G., 17

Pan, Aifei, 21

Pan, Feng, 10

Pomerantz, Michael, 08, 01

Qiao, Jie, 08

Quattrociocchi, Nick, 14

Ravindra, Deepak, OE, OF

Redien, M., 06

Rinkus, M., OB

Rojacher, Cornelia, $1 \mathrm{U}$

Roll, Christopher D., 12

Ross, James, 11, 15

Rumpel, Armin, OP

Ruppel, Thomas, OP Salsbury, Chase, 17

Schmelzer, Olaf, $\mathrm{OH}$

Schmitt, Robert Heinrich, $1 \mathrm{~V}$

Sebastian, Thomas, 12

Shahinian, Hossein, OE, OF

Sukegawa, Takashi, is

Sung, Cheng-Kuo, 20

Supranowitz, Chris, $\mathrm{OZ}$

Tayabaly, Kashmira, OM

Taylor, Lauren, 08

Thibault, Simon, 2C

Toyoshi, Yui, 11

VanKouwenberg, Jim, 04

Vogel, Paul-Alexander, IV

Vu, Anh-Tuan, 1U, 1V

Waak, Thomas, $\mathrm{OH}$

Wang, Chung-Ying, 23

Wang, Jian, OA, 10

Wang, Jung Hsing, 23

Wang, Tianyi, OM

Wang, Wenjun, 2

Wang, Zheng, 10

Watson, Stephen, 00

Wei, Yaowei, 10

Wen, Sheng-lin, OK

Wen, Sy-Bor, IP

Williamson, Ray, 1D

Wolfs, Franciscus, 11

Wu, Qian, 10

Wu, Wen-Hong, 29

Xie, Ruiqing, OA

$X \cup$, Jing, 08

Xu, Qiao, OA

Yamada, Itsunari, $1 Y$

Yeo, Woo-Jong, 26, 2E

Zaytsev, Dmytro, OE, OF

Zhang, Fei, 10

Zhang, Hongjie, IP

Zhang, Qinghua, OA

Zhao, Shijie, OA
Zheng, Nan, OK

Zhong, Bo, OK

viii

Proc. of SPIE Vol. 11175 1117501-8 


\title{
Conference Committee
}

\author{
Conference Chairs
}

Blair L. Unger, Rochester Precision Optics, LLC (United States) Jessica DeGroote Nelson, Optimax Systems, Inc. (United States)

Conference Program Committee

Thomas Battley, New York Photonics Industry Association (United States)

Michael J. Bechtold, OptiPro Systems, LLC (United States)

Rebecca Wilson Borrelli, Harris Corporation (United States) Christopher T. Cotton, ASE Sailing Inc. (United States) John P. Deegan, Rochester Precision Optics, LLC (United States) Michael A. DeMarco, QED Optics (United States) Apostolos Deslis, JENOPTIK Optical Systems, LLC (United States) Dan Gauch, Schneider Optical Machines Inc. (United States) Tom M. Godin, Satisloh North America Inc. (United States) Heidi Hofke, OptoTech Optical Machinery Inc. (United States) Dhananjay Joshi, Clemson University (United States) Jay Kumler, JENOPTIK Optical Systems, LLC (United States) Justin J. Mahanna, Universal Photonics Inc. (United States) Michael A. Marcus, Lumetrics, Inc. (United States) Michael N. Naselaris, Sydor Optics, Inc. (United States) Richard Nastasi, Universal Photonics Inc. (United States) John J. Nemechek, Metrology Concepts LLC (United States) Matthias Pfaff, OptoTech Optikmaschinen GmbH (Germany) Paul Tolley, Stretford End Solutions (United States) Martin J. Valente, Arizona Optical Systems, LLC (United States) Kirk J. Warden, LaCroix Precision Optics (United States)

Session Chairs

Plenary Session

Jessica DeGroote Nelson, Optimax Systems, Inc. (United States)

Advancements in Conventional Fabrication Methods

Blair L. Unger, Rochester Precision Optics, LLC (United States)

Integrating Automation and Lasers into Optics Fabrication

Matthew J. Brunelle, Optimax Systems, Inc. (United States)

Deterministic Processing of Optics

Dan Gauch, Schneider Optical Machines Inc. (United States) 
Deterministic Finishing Processes

Kirk J. Warden, LaCroix Precision Optics (United States)

New Advances in Optical Materials

Nicholas Bilis, Ohara Corporation (United States)

Optical Engineering

Jamie L. Ramsey, Rochester Precision Optics, LLC (United States)

Freeform Manufacturing

Scott DeFisher, OptiPro Systems, LLC (United States)

Advances in Freeform and Novel Metrology Techniques

Edward Fess, Corning Inc. (United States)

Surface Roughness and Optical Alignment

Matthias Pfaff, OptoTech Optikmaschinen GmbH (Germany)

Mid-spatial Frequency and Wavefront Error Measurement Methods

John J. Nemechek, Metrology Concepts LLC (United States)

Optical Coatings

Thomas Battley, New York Photonics Industry Association

(United States)

Structured Optical Surfaces

Dhananjay Joshi, Clemson University (United States) 